\title{
Morphology of the bicipital aponeurosis: a cadaveric study
}

\author{
S.D. Joshi, A.S. Yogesh, P.S. Mittal, S.S. Joshi \\ Department of Anatomy, Sri Aurobindo Medical College and Postgraduate Institute, Indore, India
}

[Received 17 May 2013; Accepted 2 July 2013]

The bicipital aponeurosis (BA) is a fascial expansion which arises from the tendon of biceps brachii and dissipates some of the force away from its enthesis. It helps in dual action of biceps brachii as supinator and flexor of forearm. The aim of the present work was to study the morphology of BA. Thirty cadaveric upper limbs (16 right and 14 left side limbs) were dissected and dimensions of the $B A$ were noted. The average width of aponeurosis at its commencement on the right was $15.74 \mathrm{~mm}$ while on the left it was $17.57 \mathrm{~mm}$. The average angle between tendon and aponeurosis on the right was $21.16^{\circ}$ and on the left it was $21.78^{\circ}$. The fibres from the short head of the biceps brachii contributed to the formation of proximal part of aponeurosis. Fascial sheath over the tendon of long head of biceps brachii was seen to form the distal part of the aponeurosis. In 5 cases, large fat globules were present between the sheath and the tendon. Histologically: The aponeurosis showed presence of thick collagen bundles. Fascial sheath covering the tendon of long head extended towards the aponeurosis and passed superficial to the tendon of biceps. Blood vessels and adipose tissue were found to be present between fascial sheath and the tendon. This morphological description of BA may be helpful 1) in elucidating the dynamic role that BA plays in normal functioning and 2) to the surgeons in the repair of ruptured biceps brachii tendon. (Folia Morphol 2014; 73, 1: 79-83)

Key words: biceps brachii, bicipital aponeurosis, supination, morphology

\section{INTRODUCTION}

Biceps brachii is an important muscle of anterior compartment of arm. Its short head and long head tendons form elongated bellies. The 2 bellies are closely applied and can be separated to within $7 \mathrm{~cm}$ above the elbow. The tendon of biceps brachii is attached to the rough posterior area of the radial tuberosity. From the medial border of the biceps tendon a fibrous expansion - bicipital aponeurosis (BA), is given out, which expands and merges with the deep fascia of the forearm covering the origin of the flexor muscles of forearm and gets attached to the posterior subcutaneous border of ulna. Biceps brachii is a powerful supinator as well as flexor of forearm [11].

The literature reviewed showed differing viewpoints regarding the morphology of $B A$ and the amount of contribution of fibres of short head and long head in its formation. Athwal et al. [1] reported that the BA originated from the distal part of short head of the biceps brachii in all 15 specimens studied; whereas, Eames et al. [7] stated major role of long head in the formation of BA. They have also reported the presence of 3 layers of the aponeurosis [7]. Dirim et al. [6] have reported that the BA is formed by superficial tendinous fibres arising from both muscle bellies. They have 


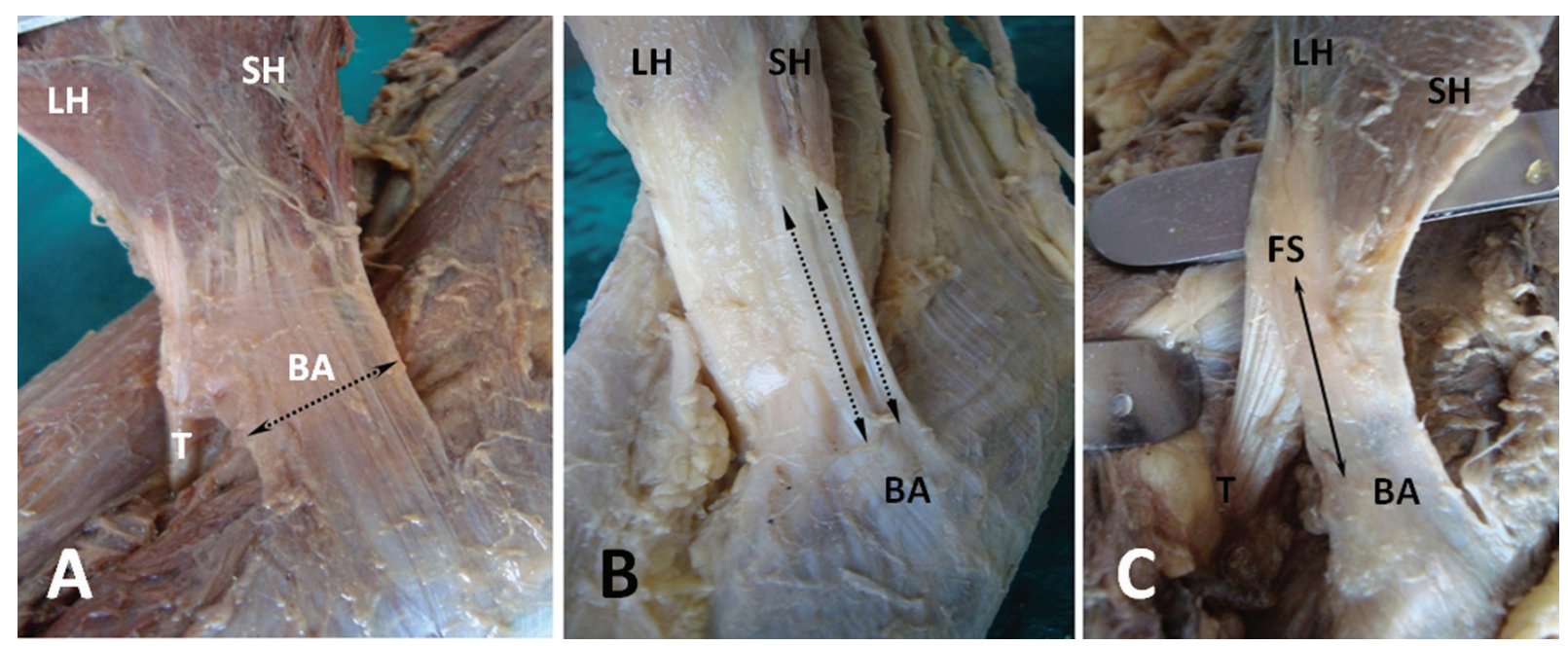

Figure 1. Formation of the bicipital aponeurosis (BA); A. Biceps brachii has been lifted from its bed to clearly show the continuity of fibres of short head (SH) in to BA; B. Direct continuation of thick tendinous fibres of $\mathrm{SH}$ (dotted double head arrows) entering and forming proximal part of BA; C. Fascial sling (FS) from the tendon of long head is seen lying superficial to the tendinous fibres of long head (LH) (double headed arrow) and forming distal part of the $\mathrm{BA} ; \mathrm{T}$ - tendon of biceps brachii.

also reported that short and long heads give varying contribution not only to the distal tendon attachment but also to the BA [6].

The role of the BA and its clinical implications are still not completely understood. The dual functional nature of biceps brachii across the elbow, i.e. flexion and supination of forearm, indicates that BA may contribute to these actions. Chew and Giuffre [4] have mentioned that in the complete tear of the biceps tendon, there is discontinuity and if the BA is also ruptured, there is retraction of the tendon. Non-consideration of anatomic details during repair of these ruptures has been reported to have a significant loss of range of supination which might be due to improper fixation of the BA to the repaired tendon [9]. There is no unanimity in the reviewed literature, concerning the role of the BA in the actions of the biceps brachii. Eames et al. in 2007 [7] have emphasized the role of long head in supination and the short head with BA in flexion, while contrary to this Dirim et al. [6] have described the role of the BA in the supination. The aim of the present study was to assess the morphology of BA and to correlate its role in the actions of biceps brachii.

\section{MATERIALS AND METHODS}

Thirty upper limbs (16 right and 14 left limbs) available in the Department of Anatomy were dissected. Skin and superficial fascia over the biceps brachii muscle was carefully removed. The BA was identified and cleaned. It was traced from its proximal to its distal attachment to the posterior subcutaneous border of ulna. The presence of muscular/tendinous fibres and the condensation of fascia were observed in the BA. The contribution from short and long head of biceps in the formation of BA was observed and recorded. The width of aponeurosis was measured at its commencement with the help of digital Vernier calliper. The angle between aponeurosis and tendon was measured at the point of divergence of the BA from the biceps brachii tendon with the help of goniometer.

Transverse sections of the tendon of biceps and BA were processed histologically to see the structure and arrangement of fibres in the BA under the light microscope after routine histological procedure. Sections 7- $\mu \mathrm{m}$-thick were cut and stained with haematoxylin and eosin and Masson's trichrome staining.

\section{RESULTS}

The BA was found to commence at the musculotendinous junction of biceps brachii. In all the specimens examined the BA received almost equal contribution from both heads of biceps brachii. The fibres from the short head constituted the proximal part of $B A$ whereas that of the long head formed the distal part of BA. In many specimens musculotendinous fibres from short head could be seen continuing in BA (Fig. 1A), whereas the contribution from long head was in the form of a thick fascia (Figs. 1B, C; $2 A, B)$. Exploring further, it was clearly seen that there was no contribution of muscular/tendinous fibres of long head to the BA. The thickened fascia could be 

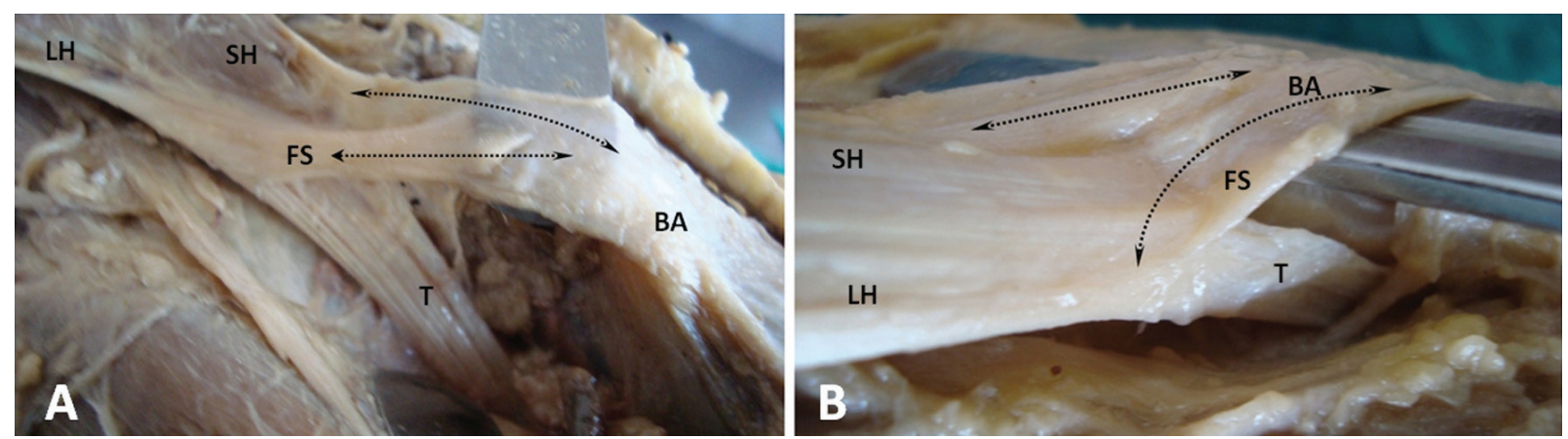

Figure 2. A, B. Contribution of long head to the bicipital aponeurosis (BA). BA has been pulled forwards to see distinctly the contributions from the two heads in the formation of BA. Fascial sling (FS) from tendon of long head (LH) joins to form distal part of the aponeurosis; $\mathrm{SH}$ - short head; T — tendon of biceps brachii.

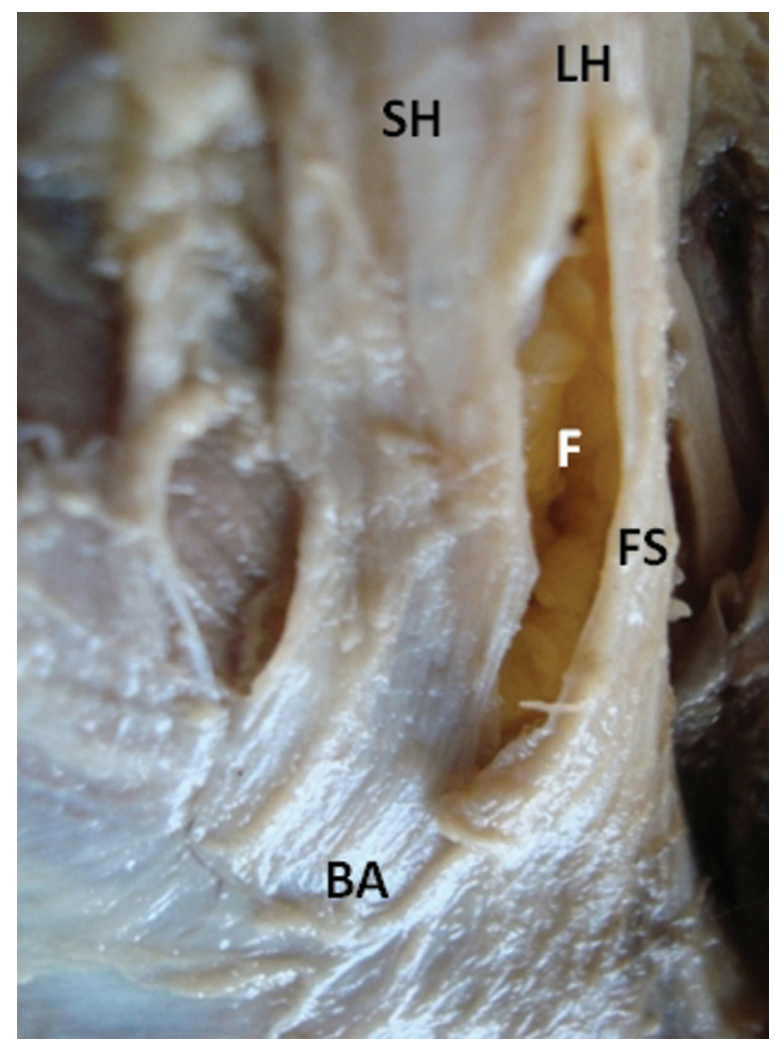

Figure 3. Pocket of fat (F) deep to the fascial sling shown after vertical incision on the sling; BA — bicipital aponeurosis; FS — fascial sling; SH — short head; LH — long head.

easily raised from the long head tendon where it was placed superficial to it and was found to be attached to the lateral margin of long head tendon (Figs. 2A, B). This fascial sheath could be slit open and in about $16 \%$ specimens it was occupied by varying amount of fat (Fig. 3).

The average width of aponeurosis at its commencement on right was $15.74 \mathrm{~mm}$ while on the left it was $17.57 \mathrm{~mm}$. The average angle between tendon and

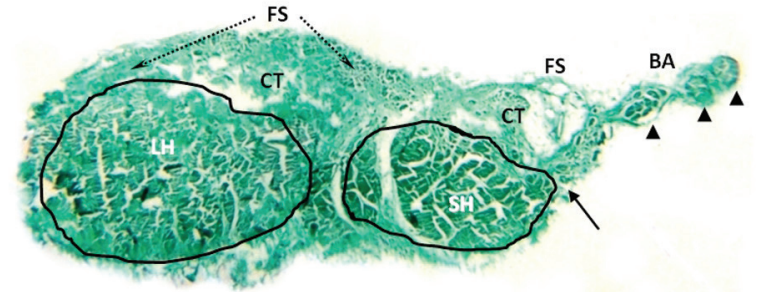

Figure 4. Transverse section through biceps brachii tendon and the bicipital aponeurosis (BA): the aponeurosis shows the presence of thick collagen bundles (arrowheads) connected with each other by connective tissue. These bundles were in continuity with collagen bundles of short head tendon (SH) (arrow). A fascial covering (FS) (dotted arrows) over the long head tendon (LH) is seen passing superficial to both the heads. Blood vessels and loose connective tissue (CT) is found to be present between FS and tendinous fibres of biceps [Masson's Trichrome stain, X10].

aponeurosis on the right was $21.16^{\circ}$ and on the left it was $21.78^{\circ}$. There was no significant difference either in the width or angle on the two sides on application of unpaired student ' $t$ ' test ( $p$ value $<0.05$ ).

Histology: Tendinous fibres of varying thickness could be seen to form the tendons of long and short heads wrapped by epitendineum (Fig. 4). On one side of the section we could clearly observe the BA in which bundles of collagen fibres could be distinctly seen. A thick connective tissue lamina was observed superficial to the tendon of long head separated by some amount of adipose and vascular tissue. Its one margin was seen to become continuous with the lateral margin of long head. Traced medially it thins out to become confluent with the BA.

\section{DISCUSSION}

The BA is a classical example of a fascial expansion which arises from a tendon and dissipates some of the load away from its enthesis. Eames et al. [7] 
Table 1. Morphology of the bicipital aponeurosis*

\begin{tabular}{lccc}
\hline Side & Number & Width of aponeurosis\# [mm] & Angle between tendon and aponeurosis^ \\
\hline Right & 16 & $15.74 \pm 5.50$ & $21.16 \pm 6.95^{\circ}$ \\
Left & 14 & $17.57 \pm 5.16$ & $21.78 \pm 6.55^{\circ}$ \\
\hline
\end{tabular}

*All values indicate mean \pm standard deviation; \#Width of the aponeurosis was measured at its commencement; $\wedge$ Angle between the aponeurosis and the tendon measured at the point of their separation

have suggested that this aponeurosis may stabilize the tendon of biceps brachii distally. In doing so, it reduces movement near the enthesis and thus stress concentration at that site.

The morphology of the BA and its applied significance have not been thoroughly investigated and appreciated till date. Athwal et al. [1] reported that the BA originates from the short head of the biceps but have not mentioned any contribution from the long head in the formation of BA. Dirim et al. [6] have reported that the BA was formed by superficial tendinous fibres arising from both short and long head. They also reported in one out of 17 cases, fibres of only the short head of the biceps brachii tendon contributing to the aponeurosis. Further, they have stated that the short and long head offer varying contribution not only to the distal tendon of biceps brachii but also to the BA [6]. Athwal et al. [1] reported that the $B A$ originated from the distal part of short head of the biceps in all 15 specimens studied; whereas, Eames et al. [7] stated major role of long head in the formation of BA. Dirim et al. [6] have reported that the $\mathrm{BA}$ is formed by superficial tendinous fibres arising from both muscle bellies. They have also reported that short and long head give varying contribution not only to the distal tendon attachment but also to the BA.

Eames et al. [7] have reported the presence of 3 layers in the BA. The superficial, thickest layer, was shown to arise from radial side of long head and crossed the short head tendon superficially. Middle layer was in the form of loose areolar tissue, which was the connection between short head and the aponeurosis. This indicates that there is no direct impact of aponeurosis on the actions of short head. Third layer arose from the deep aspect of long head from its radial side and reinforced superficial layers of the aponeurosis. There is no mention about the role of short head in the formation of the aponeurosis [7]. In the present study, there was seen direct contribution and continuation of short head fibres in the formation of proximal part of aponeurosis in all the cases studied. This finding has not been reported in the literature searched. The fascial sling found in the present study mimics the superficial layer of Eames while pocket of fat mimic his second layer. The third layer of Eames was not found in the present study.

In the present study, there was definite contribution from both the heads of biceps brachii in all the specimens examined. This is in agreement with Dirim et al. [6] and at variance with the description given by Athwal et al. [1] and Eames et al. [7]. It was observed that there was considerable contribution from the short head to the proximal part of BA and in many instances it was in the form of strong musculotendinous fibres. Such a finding has not been described in the literature. The contribution from the long head was in the form of a fascial sling which was attached to its lateral border passing superficial to the tendon and forming distal part of BA (Figs. 2A, B). In a number of cases this was considerably thick and could be easily lifted from the tendon of long head (Fig. 3). This finding is partially in agreement with that described by Eames et al. [7], but even when studied histologically it failed to show a third layer deeper to long head. Our observation that the fascial sling, which is placed superficial to long head tendon can be slit open and that there is varying amount of fat separating it from long head tendon (lubricating mechanism?) has hitherto not been described in the literature. The acute angle $\left(\approx 21^{\circ}\right)$ that was found between the biceps tendon and its aponeurosis (Table 1) has not been reported by earlier workers. This angulation may facilitate the direct transmission of forces of biceps to the ulna. Tendon of biceps brachii has both bony and fibrous attachments at its distal end. The pull of the long head of biceps is mainly concentrated on the radial tuberosity, while the pull through the $B A$ is of the fibres from the short head onto the ulna. By tensing the deep fascia, the aponeurosis increases the effectiveness of the muscle as a supinator. We hypothesized that the direction of pull of long head may be changed due to the attachment of aponeurosis to the long head of biceps brachii through the fascial 
sling. The BA being fibrous is non-elastic and when there is flexion at the elbow it will exert the pull on the tendon of biceps downwards and medially and this can help in producing strong supination by biceps during flexion at the elbow. This is besides the role played by the attachment of tendon of biceps to the posterior part of radial tuberosity.

Rupture of distal biceps tendon typically occurs in manual labourer between 30 to 50 years of age as the result of forceful extension (straightening) of the arm from initially flexed elbow position. The tendon may be completely ruptured and retracted upward leading to a noticeable bulge in the biceps muscle if the BA is also ruptured $[4,8]$. Successful surgical repair of a ruptured distal biceps tendon should be contemplated on a thorough understanding of the anatomy of BA and the tendon. Those surgical techniques which do not take into account the anatomy of aponeurosis and distal biceps tendon lead to loss of range of supination, even a decrease in supination greater than $30^{\circ}$ in some patients [5, 9]. Bain et al. [2] and Bell et al. [3] have also reported decrease in strength of supination. Chew and Giuffre [4] have shown that magnetic resonance imaging in FABS (flexed elbow, abducted shoulder, forearm supinated) position is useful in the demonstration of discontinuity of the distal tendon of biceps and intactness of BA. Thickened BA can be a causative factor for Pronator syndrome which produces the entrapment of the median nerve at the elbow. Production of pain by resistance to forearm supination and elbow flexion is considered a positive sign for median nerve entrapment by the BA [10]. By repairing a disrupted $B A$ accompanying a rupture of the distal biceps brachii tendon, the traction on the ulna can be restored [3].

\section{CONCLUSIONS}

A thorough understanding of the morphology of $B A$, including its derivation from the tendon of biceps brachii is of great value in proper appreciation of the biomechanics of action of the biceps brachii muscle at the elbow and radioulnar articulations. The present work disagrees with the findings of many earlier workers and shows that upper proximal part of BA is mainly contributed by fibres from short head of biceps brachii; whereas the distal part of BA is primarily a fascial structure which is attached to the radial margin of the long head tendon passing superficial to this tendon. This fascial sling seems to be a mechanism of nature to keep the tendon of biceps angulated during flexion so that it can work efficiently as a supinator, while the 2 heads together can bring about flexion at the elbow joint.

\section{REFERENCES}

1. Athwal GS, Steinmann SP, Rispoli DM (2007) The distal biceps tendon: footprint and relevant clinical anatomy. J Hand Surg Am, 32: 1225-1229.

2. Bain GI, Prem H, Heptinstall RJ, Verhellen R, Paix D (2000) Repair of distal biceps tendon rupture: a new technique using the endobutton. J Shoulder Elbow Surg, 9: 120-126.

3. Bell HR, Wiley WB, Noble JS, Kuczynski DJ (2000) Repair of distal biceps tendon ruptures. J Shoulder Elbow Surg, 9: 223-226.

4. Chew ML, Giuffre BM (2005) Disorders of the distal biceps brachii tendon. Radiographics, 25: 1227-1237.

5. Davison BL, Engber WD, Tigert $\amalg$ (1996) Long term evaluation of repaired distal biceps brachii tendon rupture. Clin Orthop Relat Res, 333: 186-191.

6. Dirim B, Brouha SS, Pretterklieber ML, Wolff KS, Frank A, Pathria MN, Chung CB (2008) Terminal bifurcation of the biceps brachii muscle and tendon: anatomic considerations and clinical implications. Am J Roentgenol, 191: W248-W255.

7. Eames MH, Bain Gl, Fogg QA, van Riet RP (2007) Distal biceps tendon anatomy: a cadaveric study. J Bone Joint Surg Am, 89: 1044-1049.

8. Hearon BF (2008) Distal biceps repair. Available at http:// //www.drhearon.com/WTL\%20December\%2008.htm.

9. Kulshreshtha R, Singh R, Sinha J, Hall S (2007) Anatomy of the distal biceps brachii tendon and its clinical relevance. Clin Orthop Relat Res, 456: 117-120.

10. Martinelli P, Gabellini AS, Poppi M, Gallassi R, Pozzati E (1982) Pronator syndrome due to thickened bicipital aponeurosis. J Neurol Neurosurg Psychiatry, 45: 181-182.

11. Standring $S$ (2008) Gray's anatomy. $40^{\text {th }}$ Ed. Churchill Livingstone Elsevier, Edinburgh, pp. 825-826. 